\title{
mécanique des roches \\ et évacuation des déchets radioactifs \\ en formations géologiques
}

par

B. Côme

C. C. E., Bruxelles

\section{Introduction}

Les déchets de haute activité provenant de l industrie nucléaire posent un problème dont l'acuité croît avec le développement de l'énergie électronucléaire dans les pays industrialisés. Ces déchets doivent être isolés de l'environnement humain pendant une très longue durée, pour garantir que leur retour dans la biosphère ne présente aucun danger pour cet environnement. L'utilisation d'un système de confinement associant plusieurs enveloppes, appelées "barrières artificielles" (ex. : conteneur métallique), et l enfouissement profond dans des formations géologiques adéquates est considéré comme un moyen pratique, et économiquement satisfaisant, d'atteindre ce but.

Les propriétés de confinement de la roche-hôte doivent subsister pendant très longtemps. II apparaît donc nécessaire d'acquérir le plus rapidement possible une bonne connaissance des propriétés des formations et, surtout, de leur évolution au cours du temps et sous l'effet des sollicitations nouvelles introduites par l'évacuation des déchets elle-même.

\section{Le programme de la commission des Commu- nautés européennes}

La commission des Communautés européennes contribue depuis 1975 à cet effort d'approfondissement des connaissances dans le cadre de ses programmes quinquennaux de recherche et de développement, sous forme de contrats de recherche à frais partagés, dans le domaine de la gestion et du stockage des déchets radioactifs. Un des composants de ce programme, appelé "Stockage et évacuation en formation géologique", regroupe et coordonne un grand nombre de travaux de recherche effectués dans des organisations des États-membres et au Centre commun de recherches d'Ispra (Italie). Ces travaux visent à acquérir une meilleure connaissance des formations rocheuses et de leur évolution.

Pour des raisons en partie historiques, l'effort de recherche a été concentré sur trois types de formations considérées dès le départ comme favorables à l'évacuation des déchets radioactifs :

- Les roches granitiques, envisagées pour leur résistance mécanique, leur stabilité et leur faible perméabilité. Les travaux les concernant sont effectués principalement en France et au Royaume-Uni.

- Les formations argileuses, retenues à cause de leur capacité de fixation des radionucléides éventuellement libérés par les déchets enfouis, et aussi pour leur imperméabilité, étudiées en Belgique, en Italie et récemment au Royaume-Uni.

- Les formations salines, essentiellement pour leur très faible teneur en fluides et pour leur perméabilité à peu près nulle. Les Pays-Bas et l'Allemagne Fédérale concentrent leurs efforts sur ce type de roche (sel en dôme ou en couches).

D'un point de vue pratique, les sujets de recherche sont organisés dans le cadre d'une "classification opérationnelle " détaillée dans le tableau 1.

On constate que la mécanique des roches est largement représentée dans ces travaux (sujets 1,2 , $3,5,6,8,9$ essentiellement). Les thèmes concernés peuvent se regrouper, selon une logique plus * scientifique $*$, en quatre grandes rubriques :

1) Investigation " géodynamique * des sites potentiels.

2) Mesure et modélisation des propriétés mécaniques des roches à l'échelle de l'échantillon.

3) Mesure et modélisation des propriétés et du comportement mécaniques à grande échelle des massifs rocheux.

4) Construction des dépôts profonds définitifs : génie civil, génie minier, etc.

Les paragraphes qui suivent procèdent à une illustration de ces quatre rubriques, à partir d'exemples 
empruntés aux contrats en cours dans la période 1980-1982 (phase 1) du deuxième (et actuel) programme quinquennal de recherche et développement. Ce programme couvre la période 1980-1984.

Ils montrent également les progrès réalisés en mécanique des roches suite à l' "éclairage " très particulier résultant de l'aspect "évacuation de déchets radioactifs" et des contraintes qui s'y associent.

Remarque: II est bien évident que le découpage séquentiel en quatre thèmes recouvre en fait de nombreuses interactions et rétroactions; il n'a été adopté ici que pour la commodité de l'exposé.

Tableau 1

Classification "opérationnelle des travaux de $R$ et $D$ du Programme

"Stockage et évacuation en formation géologique *

I. STOCKAGE ET ÉVACUATION EN FORMATIONS "CONTINENTALES $*$

1. Sélection des sites. Caractéristiques générales.

2. Programmes de forages profonds.

3. Puits et chambres expérimentales souterraines.

4. Barrières artificielles:

4.1. Conteneur pour déchets.

4.2. Barrières géochimiques, colmatage et scellement.

5. Équilibres internes des formations, avant et après évacuation :

5.1. Caractéristiques des formations.

5.2. Migration des radionuclides.

6. Modèles mathématiques.

7. Analyses de sûreté.

8. Installations-pilote d'évacuation.

9. Mise au point de techniques de construction et d'exploitation.

10. Conception d'installation d'évacuation.

II. ÉVACUATION DANS LES FONDS MARINS

III. AMÉLIORATION DES TECHNIQUES

D'INVESTIGATIONS

1. Études fondamentales d'intérêt général.

2. Mise au point de méthodes de mesure.

\section{Exemples de progrès en mécanique des roches}

\section{Investigation "géodynamique" de sites potentiels}

Comme il a été expliqué précédemment, les formations géologiques susceptibles d'accueillir des déchets radioactifs ont été choisies à l'origine pour leur stabilité sur de très longues durées, comparables à celles des périodes géologiques. II est cependant reconnu que les formations géologiques évoluent, plus ou moins lentement, sous l'effet de facteurs qui relèvent :

- de la géodynamique dite "interne ": parmi ceux$\mathrm{ci}$, on peut inclure le volcanisme, la fréquence et l'action de failles et fractures, la séismicité, et le diapirisme (spécifique des dômes de sel);

- de la géodynamique dite "externe»: la cli- matologie, l'érosion et la sédimentation sont les plus notables de ces facteurs.

Les études de sûreté relatives à l'évacuation de déchets radioactifs dans un site donné nécessitent une prédiction, la plus fiable possible, du devenir de la formation géologique considérée. II est clair que cette prédiction sera d'autant plus réaliste que l'évolution antérieure du site aura été connue sur une période plus longue dans le passé.

C'est ainsi que l'analyse géodynamique rétrospective (historique) d'un site donné permet d'accéder à un modèle géodynamique "prospectif s de ce site. Pour fixer les idées, on peut admettre qu'une prédiction fiable sur un million d'années environ nécessite la connaissance détaillée de l'histoire géodynamique antérieure du site sur tout le quaternaire $(2,5$ à 3 millions d'années). II est bien entendu que l'analyse ne concerne pas seulement les facteurs géodynamiques cités ci-dessus isolément, mais encore et surtout le jeu complet de leurs interactions. En conséquence, les exigences inhabituelles de prédiction d'un site rendent nécessaire une connaissance du passé de ce site bien plus approfondie que pour des ouvrages ordinaires de génie civil par exemple, et surtout non limitée à la simple géologie.

Actuellement, cette approche est appliquée à certaines unités géologiques en France. On peut espérer qu'à terme elle fournira le cadre général pour des études de détail qu'on va maintenant envisager.

\section{Mesure et modélisation des propriétés mécaniques des roches à l'échelle de l'échantillon}

Des échantillons de roches provenant de divers sites de la Communauté sont étudiés au laboratoire sous des conditions variées de contrainte et de température. Un effort important est accordé sur ce dernier type de sollicitation. Le dégagement de chaleur est en effet une caractéristique des déchets de haute activité que l'on envisage d'enfouir à grande profondeur; les effets de l'échauffement se feront surtout sentir pendant les premières décennies suivant l'évacuation, et dans ce que l'on appelle le "champ proche du dépôt.

\subsection{Granite}

Vu les difficultés expérimentales, les essais réalisés à ce jour l'ont été sur des échantillons soumis à un certain échauffement puis refroidis avant chargement. On peut ainsí déterminer l'effet,.sur les propriétés mécaniques classiques, d'un traitement thermique donné.

A partir des expériences réalisées en France et au Royaume-Uni sur des granites d'origines différentes, on peut mettre en évidence un certain nombre de conclusions générales.

La durée et l'intensité du chauffage ont toutes deux une influence sur les propriétés mécaniques. La résistance (en compression simple par exemple) et la déformabilité sont systématiquement affectées par le développement d'une microfissuration du granite, due à la différence des coefficients de dilatation de ses composants. Cette microfissuration est mise en évidence par :

- augmentation de la porosité après chauffage : les mesures au porosimètre et celles déduites de la 


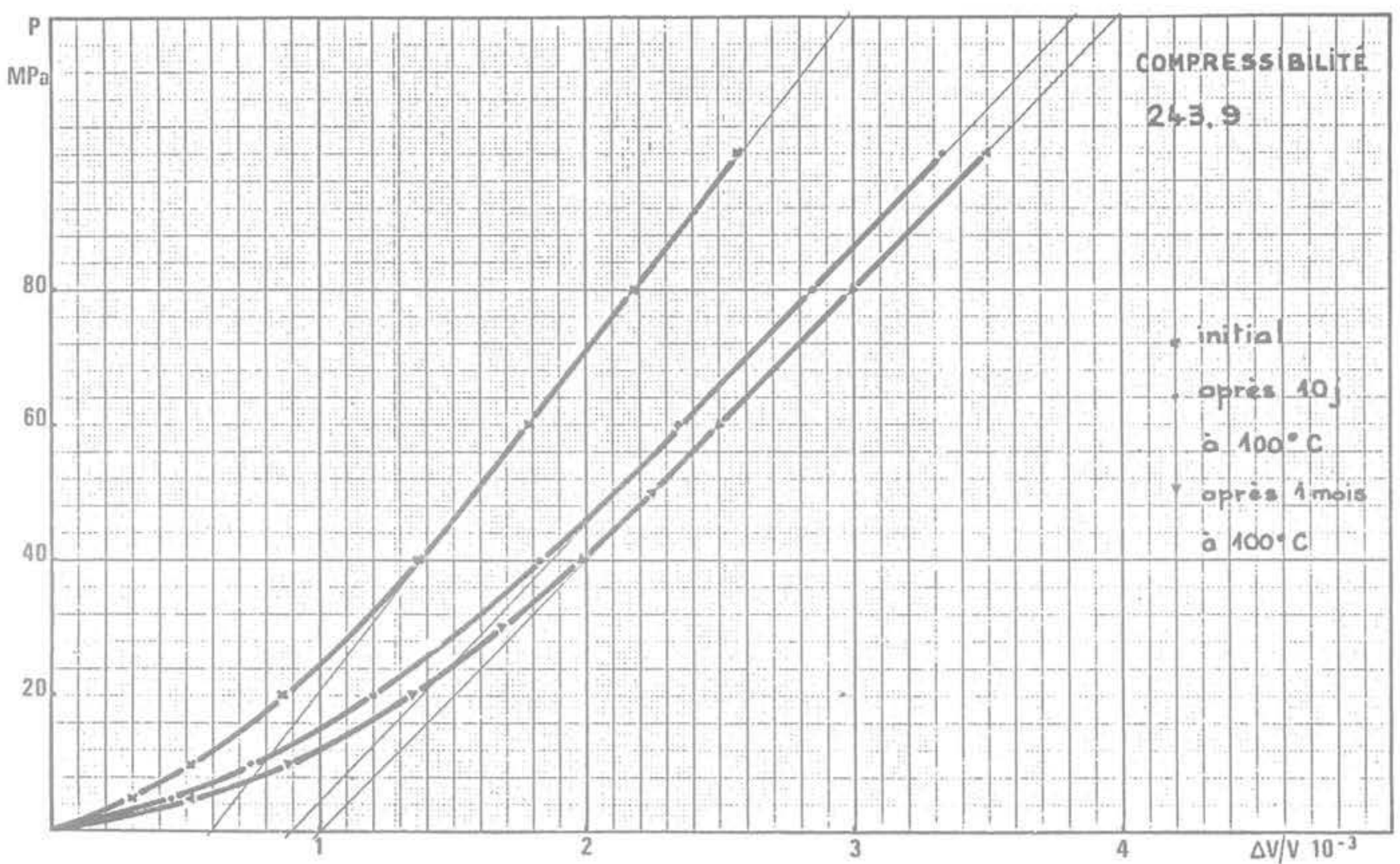

Fig. 1 Evolution de la compresibilité d'un granite en fonction de la durée du chauffage

Tableau 2

\begin{tabular}{c|c|c|c}
\hline Conditions d'essai & $\mathrm{K}$ & $\mathrm{D}$ & $\mathrm{Pf}$ \\
\hline État naturel & 51 & 0,55 & 50 \\
\hline $\begin{array}{c}\text { Après } 10 \text { jours } \\
\text { a } 100^{\circ} \mathrm{C}\end{array}$ & 41,5 & 0,75 & 55 \\
\hline $\begin{array}{c}\text { Après } 1 \text { mois } \\
\text { a } 100^{\circ} \mathrm{C}\end{array}$ & 43 & 0,95 & 60 \\
\hline
\end{tabular}

compressibilité sont convergentes;

- diminution du module d'Young et augmentation du coefficient de Poisson;

- diminution de la résistance en compression simple.

A noter que ces effets sont surtout marqués au-delà d'un certain "seuil" de température qui dépend du granite considéré. Les tableaux 2 et 3 , ainsi que la figure 1, illustrent quantitativement ce qui précède.

Évolution des propriétés mécaniques d'un granite en fonction de la durée du chauffage :

- K, module de compressibilité (GPa);

- D, porosité de fissures:

- Pf, pression de serrage (MPa).

Évolution des propriétés de déformabilité et de résistance d'un granite en fonction de la durée du chauffage :

- E, module d'Young (GPa);

- $v$, coefficient de Poisson;

- $\sigma$, résistance en compression simple (MPa).
Tableau 3

\begin{tabular}{c|c|c|c}
\hline Conditions d'essai & $\mathrm{E}$ & $\boldsymbol{v}$ & $\sigma$ \\
\hline État naturel & 68 & 0,27 & 165 \\
\hline $\begin{array}{c}\text { Après } 8 \text { jours } \\
\text { à } 100^{\circ} \mathrm{C}\end{array}$ & 66 & 0,17 & 168 \\
\hline $\begin{array}{c}\text { Après } 1 \text { mois } \\
\text { a } 100^{\circ} \mathrm{C}\end{array}$ & 55 & 0,15 & 155 \\
\hline
\end{tabular}

\section{$2.2 \mathrm{Sel}$}

L'influence de la température sur le comportement caractéristique du sel, son * fluage " sous contrainte, est déjà connue.

Un résultat intéressant concerne l'influence de l'interaction entre les fluides et le sel sur sa rhéologie. En général, la teneur en fluides du sel est très faible; ceux-ci sont répartis dans des inclusions intracristallines, ou à la limite de cristaux. On peut imaginer par contre des scénarios selon lesquels un " accident " provoque l'entrée d'eau au voisinage de (ou dans) un dépôt de déchets. Cette eau se sature progressivement en sel et peut interagir avec la roche, surtout si un excès de contraintes dans le sel cause une augmentation de sa fissuration, permettant ainsi une sorte d'"imprégnation" du sel solide par de la saumure.

Des essais où la pression et la nature du fluide sont contrôlés montrent qu'il peut y avoir une forte influence de ce fluide sur la vitesse de fluage du sel. La figure 2 donne un exemple d'une telle interaction 


\section{TIME (hours)}

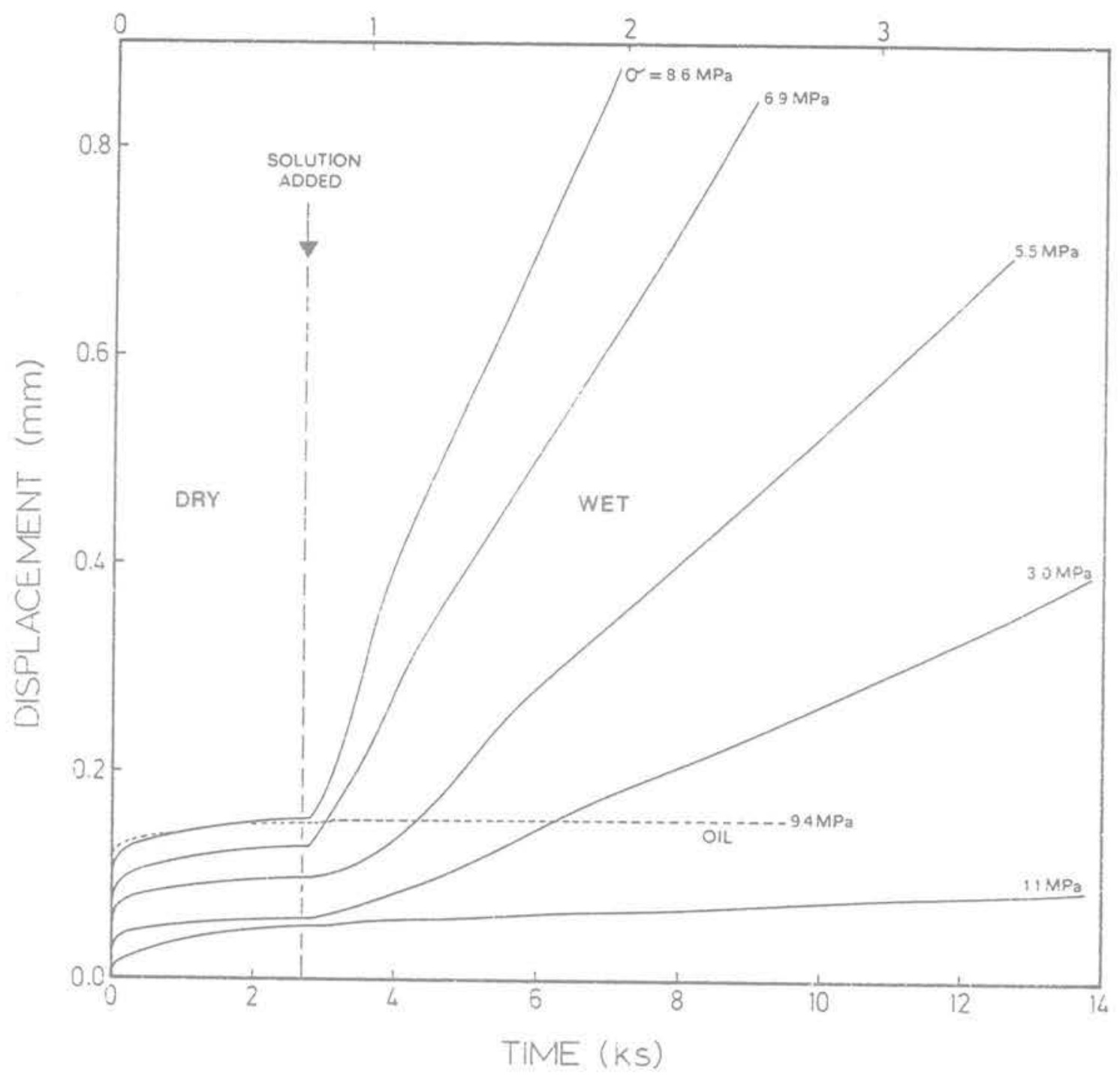

Fig. 2 Influence de l'adjonction d'une solution saturée de $\mathrm{NaCl}$ sur le fluage du sel len tireté, pour la comparaison, influence d'une adjonction de pétrole volatill.

La solution est ajoutée $45 \mathrm{mn}$ après le début du chargement à sec.

entre du sel et sa saumure saturée; l'appareillage utilisé est décrit sur la figure 3. Pour l'instant, il serait prématuré de tirer des conclusions de ce phénomène en vue de la sûreté d'un dépôt dans le sel; néanmoins, le fait mérite d'être gardé à l'esprit.

\section{Mesure et modélisation des propriétés et du comportement à grande échelle des massifs rocheux}

De nombreux essais "en place "sont actuellement effectués dans des "laboratoires souterrains" plus ou moins profonds, qui peuvent être des excavations existantes aménagées, ou des cavités spécialement créées dans ce but. Parfois aussi, certains phénomènes naturels «à grande échelle * sont directement observables sans travaux particuliers.

\subsection{Formations salines}

Une des tentatives les plus originales est celle de la mesure des contraintes en place (d'origine) dans une formation saline, à une certaine distance d'une mine existante. La méthode utilisée est celle de la fracturation hydraulique (fig. 4) dans des forages exécutés à partir de la mine, vers $800 \mathrm{~m}$ de profondeur environ. A cette date, il semble que seule cette méthode ait donné des résultats exploitables (fig, 5 et 6).

II faut cependant considérer comme préliminaires les conclusions de ces mesures, résumées dans le tableau 4 :

- existence d'un champ de contrainte anisotrope dans le sel (la majeure, de $20 \mathrm{MPa}$, étant grossièrement horizontale):

- contrainte verticale (12 MPa) inférieure au poids des terres à cette cote.

Si ces résultats se confirmaient, ils pourraient remettre en cause l'hypothèse ordinaire de l'isotropie des contraintes au moins dans certaines formations salines. Une étape supplémentaire serait de relier ce phénomène à l'histoire de la formation saline concernée; on se retrouve ainsi dans l'analyse "géodynamique " évoquée précédemment. 


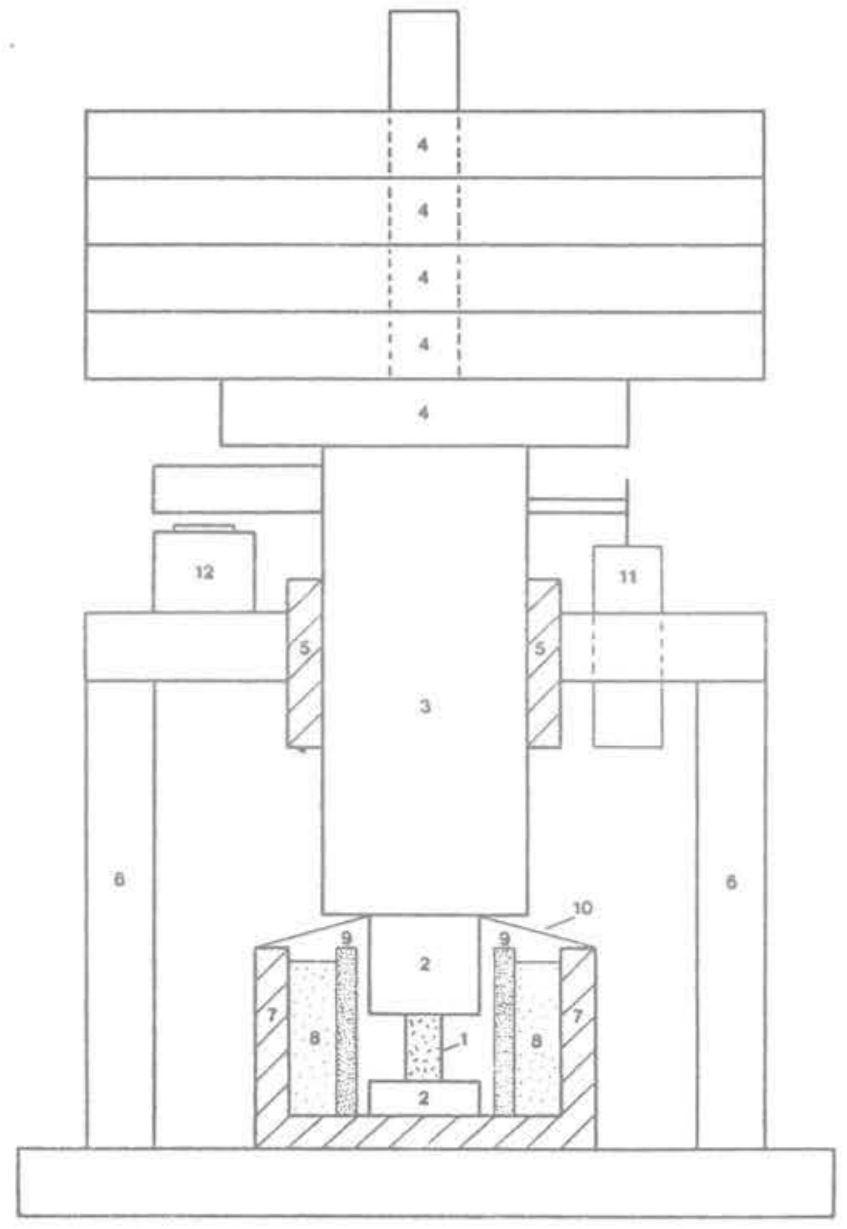

Fig. 3 Schéma de l'appareillage de fluage "poids mort» utilisé pour l'essai de la fig. 2

(1) échantillon de sel

(2) et (3) piston

(4) poids morts exerçant la charge

(5) joint à faible frottement

(6) bâti de la presse

(7) pot en plexiglas

(8) poudre de sel

(9) filtre en nylon

(10) couvercle souple

(11) capteur de déplacement

(12) vérin hydraulique

Tableau 4

\begin{tabular}{c|c|c}
\hline $\begin{array}{c}\text { Distance à la } \\
\text { galerie }(\mathrm{m})\end{array}$ & $\begin{array}{c}\text { Angle du plan } \\
\text { de fracture avec } \\
\text { la verticale }\left(^{\circ}\right)\end{array}$ & $\begin{array}{c}\text { Pendage } \\
\text { du forage }\left(^{\circ}\right)\end{array}$ \\
\hline 10,2 & 53 & 1,5 \\
18,5 & 76,5 & 2 \\
27,5 & 89,7 & 2 \\
\hline
\end{tabular}

Caractéristiques de fractures obtenues dans 3 essais de fracturation hydraulique (voir aussi fig. 6).

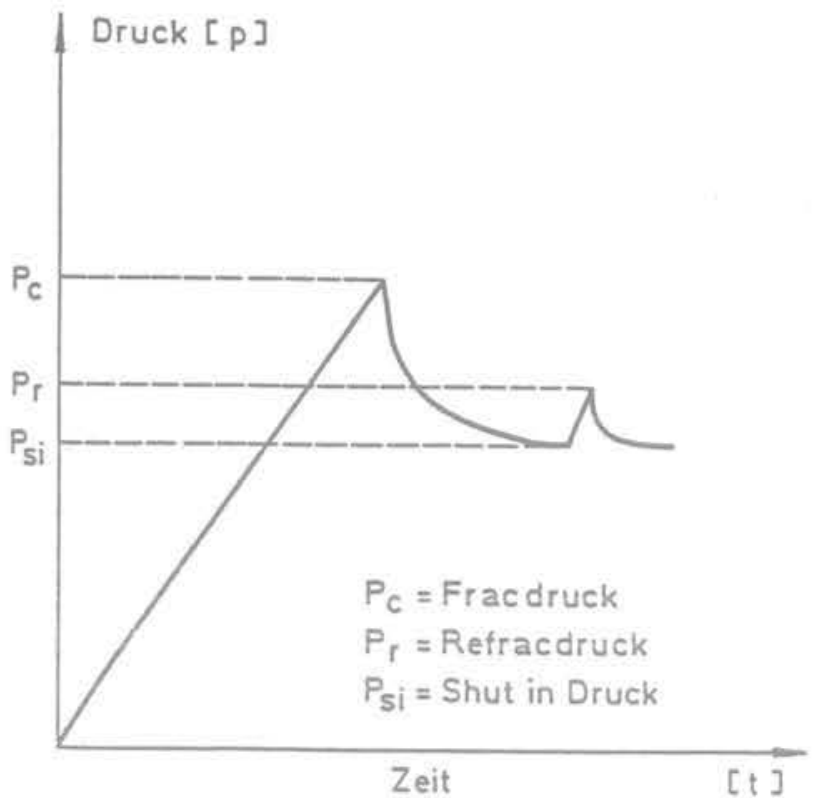

Fig. 4 Courbe schématique indiquant les grandeurs accessibles lors d'un essai de fracturation hydraulique

\subsection{Formations argileuses}

Une géologie favorable permet d'étudier deux configurations particulièrement inhabituelles dans ce type de roche.

Dans l'un de ces sites naturels (Orciatico, Toscane), des instrusions volcaniques récentes (fin du Tertiaire) ont progressé à travers un bassin d'argile tertiaire (Pliocène) provoquant en leur voisinage un métamorphisme de contact dans l'argile. Les variations de propriétés physique et mécanique de cette argile sous l'effet du chauffage et du refroidissement sont en cours d'étude.

Dans le deuxième, une carrière d'argile à ciel ouvert permet d'observer une faille traversant la couche d'argile (fig. 7), ayant affecté en premier le soubassement plus rigide. Il semblerait donc que ce genre de déplacement tectonique puisse affecter aussi certaines formations argileuses. L'élucidation du mécanisme de cette faille met en jeu des essais sur maquettes (fig. 8) et des calculs de mécanique des roches (fig. 9 et 10); les travaux devraient permettre de mieux quantifier les effets d'une faille intersectant un dépôt de déchets dans l'argile, longtemps après fermeture de ce dernier. 


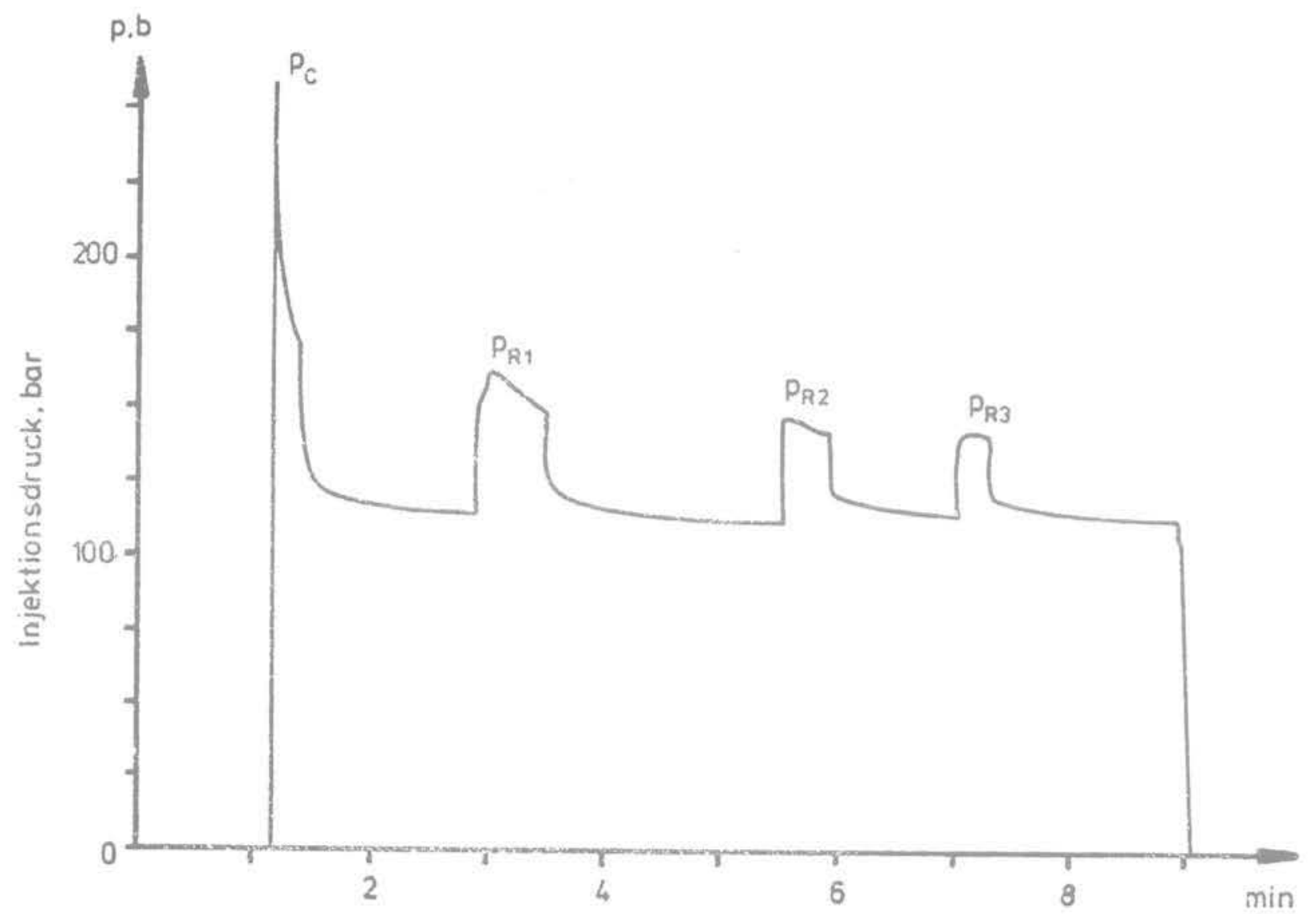

Fig. 5 Exemple de courbe de mesure obtenue lors d'un essai de fracturation hydraulique dans le sel

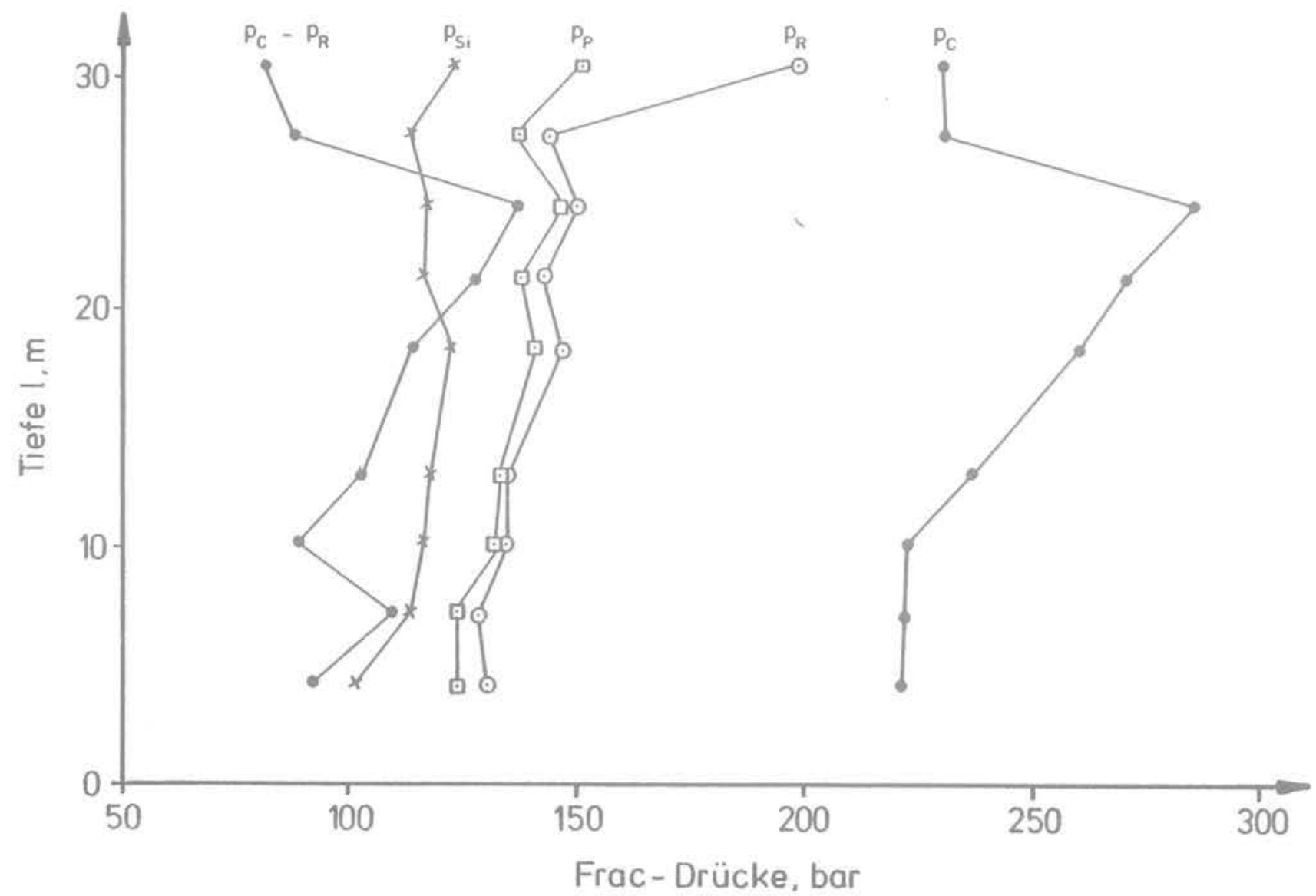

Fig. 6 Exemples de pressions de fracturation en fonction de la distance à la galerie de mine 


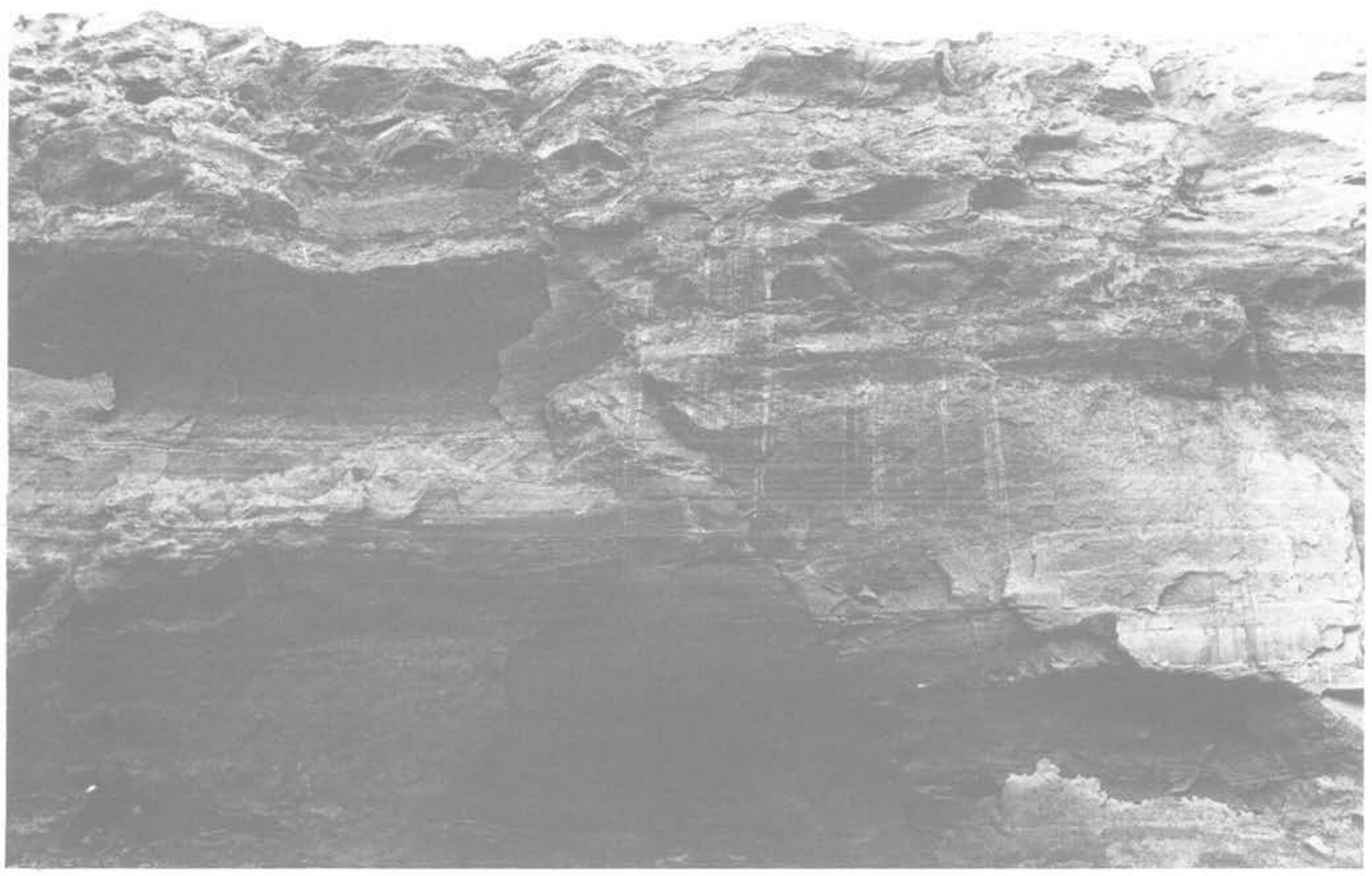

Fig. 7 Vue d'une faille au flanc d'une carrière d'argile

\section{VERTICAL RISPLEEMENT PAULT}

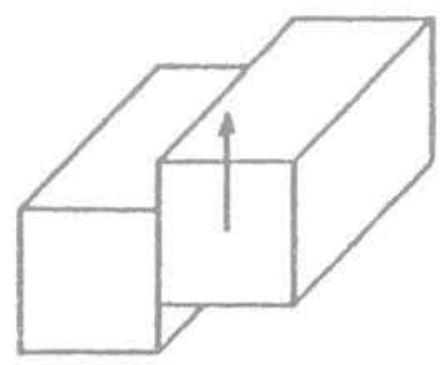

Fig. 8 Schématisation d'un mode d'action d'une faille : déplacement vertical 


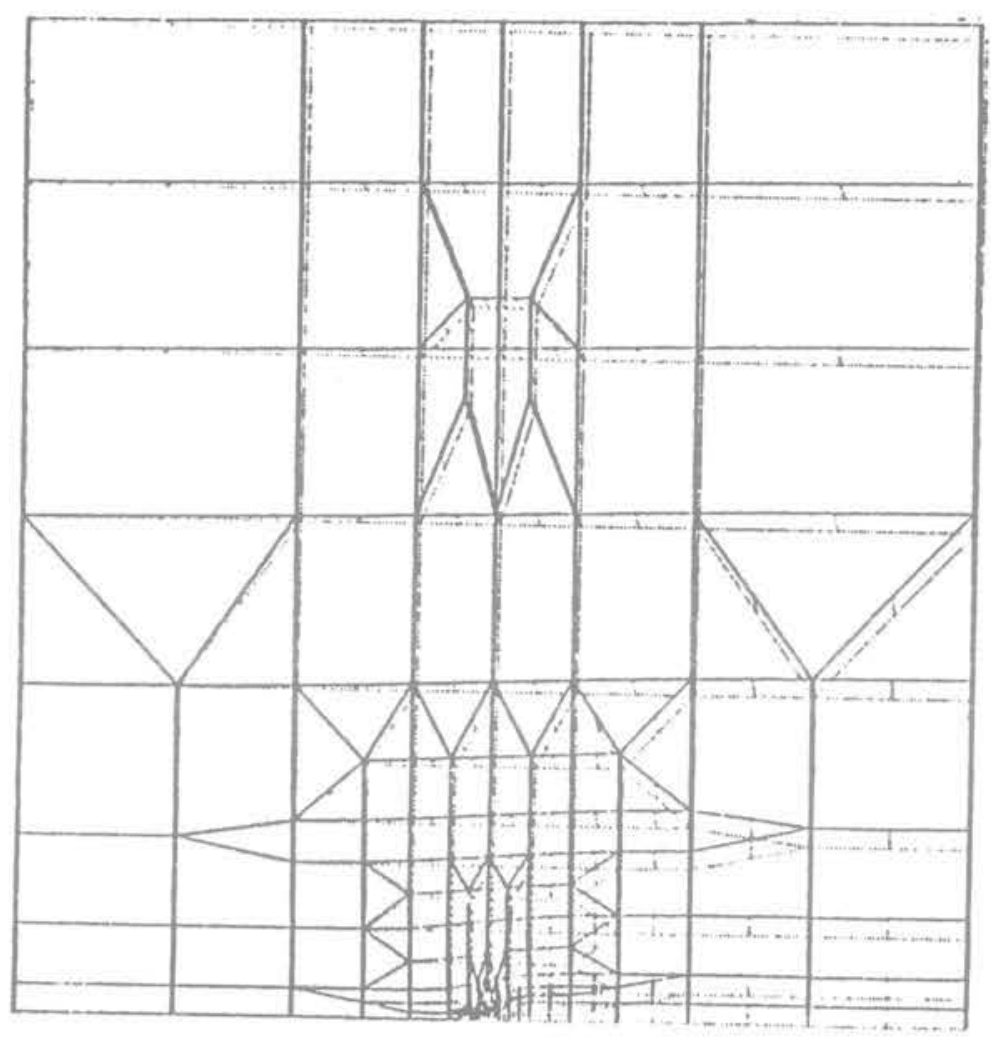

Fig. 9 Exemple de maillage d'éléments finis utilisés pour la modélisation d'une faille : - en traits pointillés : position initiale du massif

- en traits continus : position du massif après déformation

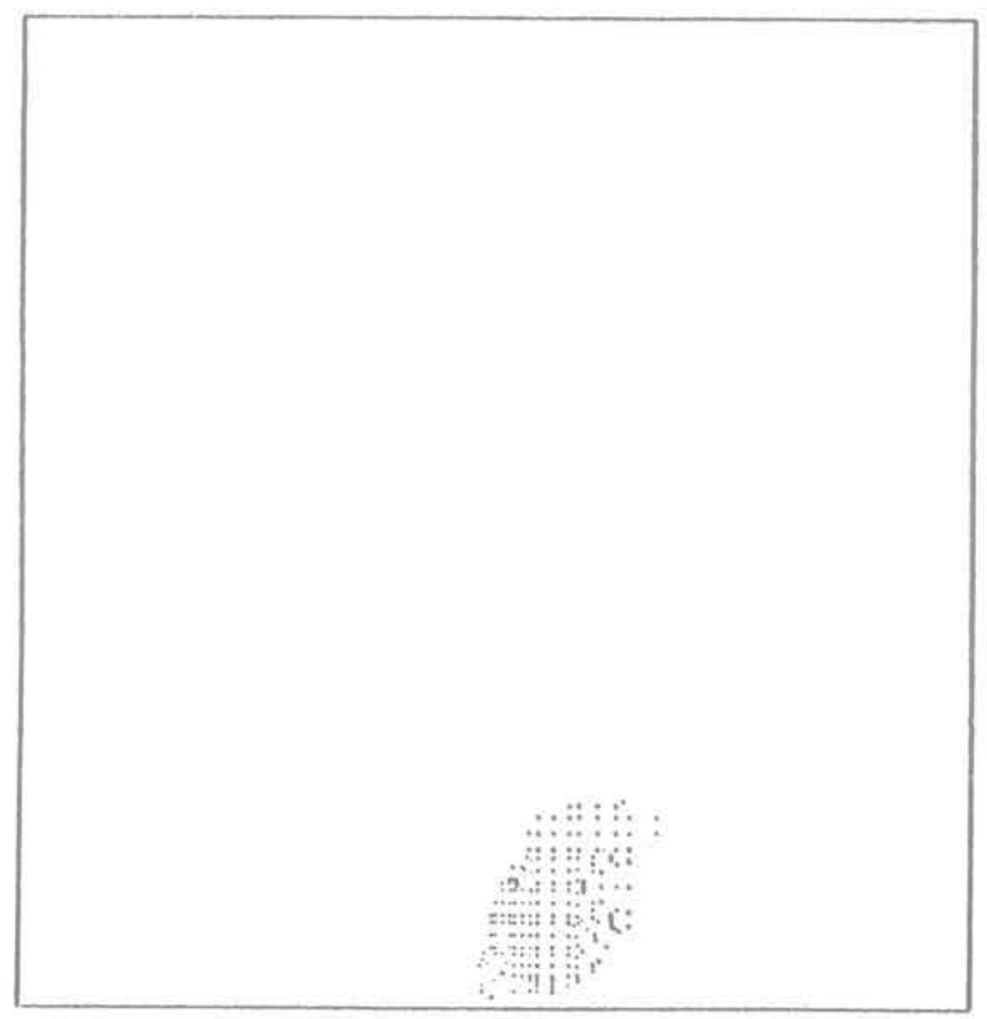

Fig. 10 Résultat du calcul de la fig. 9 sur la propagation d'une faille : extension de la zone de rupture suite au déplacement du soćle 


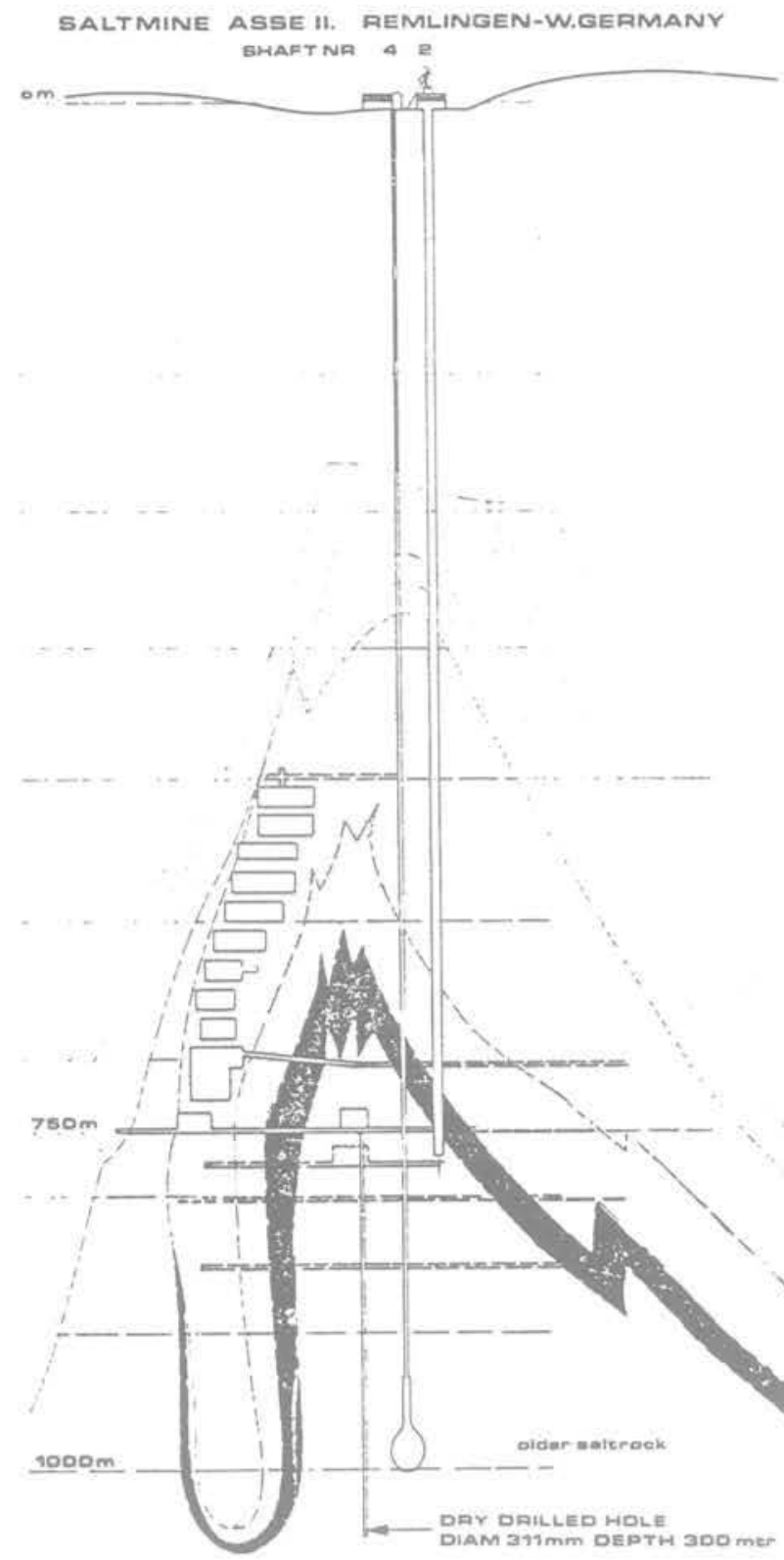

$1100 \mathrm{~m}$

Fig. 11 Coupe schématique de la mine de Asse (République Fédérale d'Allemagnel montrant l'emplacement du forage de $300 \mathrm{~m}$

\section{Construction de dépôts profonds définitifs : génie civil, minier, etc.}

De nombreuses études de conception pour de tels dépôts ont déjà été réalisées, et d'autres sont encore prévues. Actuellement, l'accord semble se faire sur deux types principaux de dépôts :

- le système "forages profonds" à partir de la surface du sol. Leur diamètre peut atteindre $50 \mathrm{~cm}$ pour une profondeur excédant le millier de mètres;

- le système des galeries souterraines horizontales minées, avec puits d'accès foncés à partir de la surface.

On peut envisager évidemment des combinaisons de ces deux systèmes. C'est ainsi que, dans le cadre d'un contrat communautaire, il a été procédé à la réalisation à $\mathrm{sec}$, dans le sel, d'un forage vertical de $300 \mathrm{~m}$ à partir d'une galerie de mine située également dans le sel, à $750 \mathrm{~m}$ de profondeur environ dans la

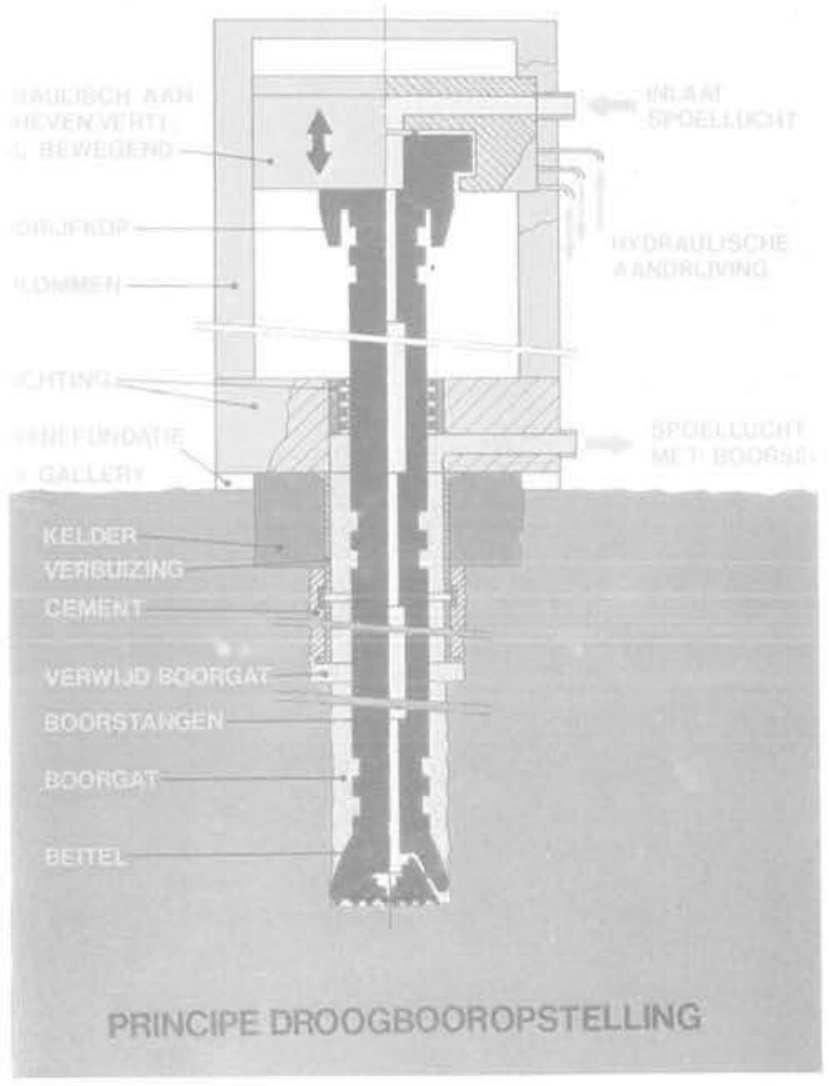

Fig. 12 Schéma de principe de la machine de forage à sec dans le sel

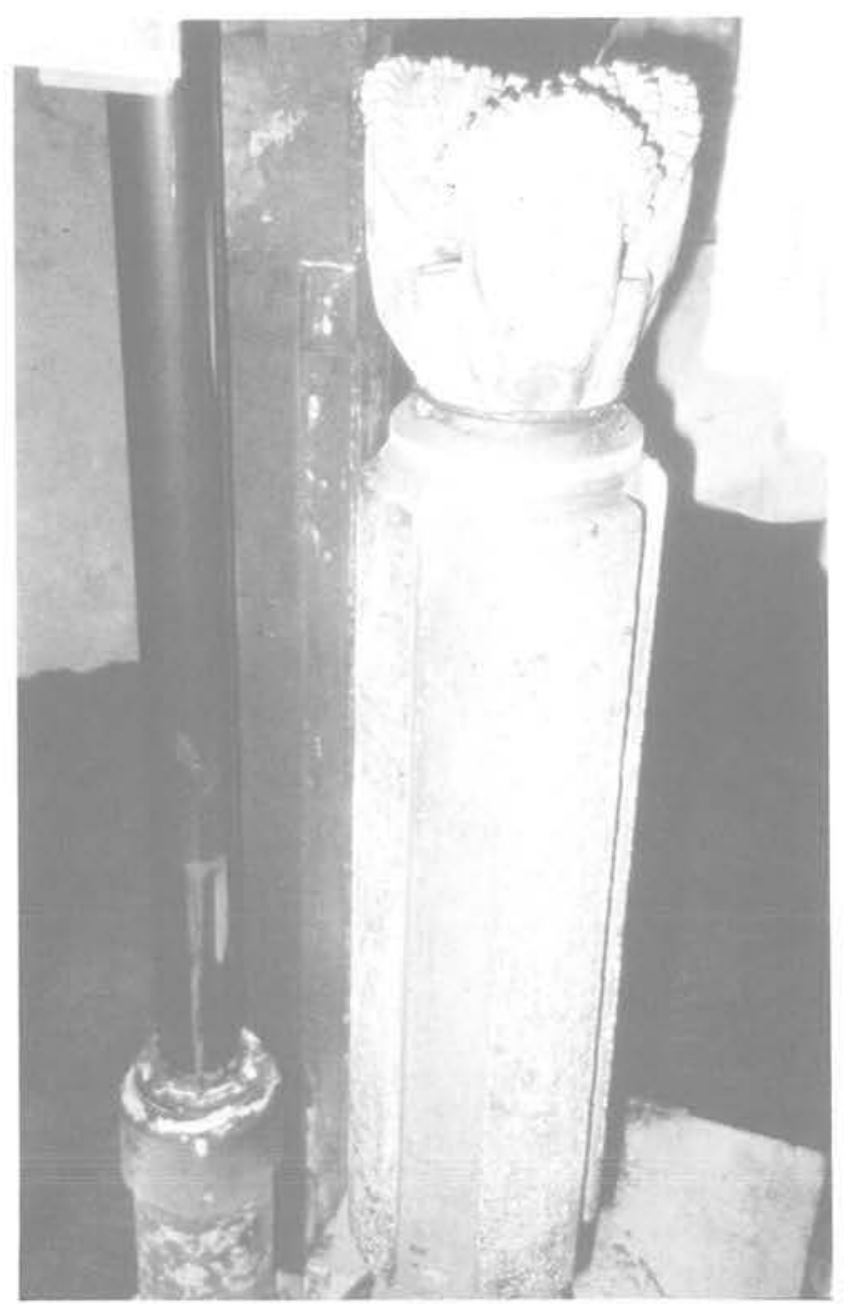

Fig. 13 Vue de l'outil de forage à sec dans le sel 
mine de Asse (République Fédérale d'Allemagne) (fig. 11): Le diamètre du forage ètait de $30 \mathrm{~cm}$ environ et la vitesse moyenne de pénétration, environ $1 \mathrm{~m} / \mathrm{h}$. Les figures 12 et 13 illustrent la technique du forage, et l'appareillage utilisé.

II est ainsi prouvé que les solutions techniques existent dès maintenant, pour remplir les exigences dérivant de la conception des dépôts de déchets radioactifs de haute activité.

\section{Conclusion}

Grâce aux acquis nouveaux dans le domaine de la mécanique des roches, dont cet exposé, volontairement bref, n'a donné qu un apercu limité, la connaissance des propriétés et du comportement des forma- tions rocheuses a grandement progressé. L'introduction des méthodes de "géodynamique prospective" permettra de plus - mais pas dans l'immédiat - de prédire, avec le maximum de confiance, l'évolution même à très long terme des sites de dépôt. L'évaluation de la sûreté des systèmes d'évacuation de déchets radioactifs en formations géologiques se fera donc sur des bases expérimentales de plus en plus fermes.

\section{Référence bibliographique}

«Radioactive Waste Management and Disposal Proceedings of the First European Community Conference $"$, Luxembourg, May 20-23, 1980. Harwood Academic Publishers, 1981.
Traduction des légendes des figures

Fig. 2

Displacement

Time

Solution added

Dry

Wet

Oil

Fig. 4

Druck

Fracdruck

Refracdruck

Shut in druck

Zeit

Fig. 5

Injecktionsdruck

Fig. 6

Tiefe

Frac-Drücke

Fig. 8

Vertical

displacement fault
Déplacement

Temps

Solution saline ajoutée

Sec

Humide

Pétrole

Pression

Pression de fracturation

Pression de re-fracturation

Pression de fermeture

Temps

Pression d'injection

Distance à la galerie

Pression à la fracturation

Faille

à déplacement vertical
Fig. 11

Salt Mine Asse II -

Remiingen -

W. Germany

Shaft Nr 4.2

Older salt rock

Dry-drilled hole,

diam. $311 \mathrm{~mm}$,

depth $300 \mathrm{mtr}$

Fig. 12

Hydraulisch aangedreven, Accouplement hydraulique verticaal bewegend juk

Aandrijfkop

4 kolomnen

Afdichting

Machine fundatie

$750 \mathrm{~m}$ gallery

Kelder

Verbuizing

Cement

Verwijd boorgat

Boorgat

Beitel

Inlaat spoellucht

Hydraulische aandrijving

Spoellucht met boorsel

Principe

droogbooropstelling
Mine de sel de Asse 11 Remlingen -

Allemagne Fédérale

Puits $n^{\circ} 4.2$

Sel «ancien»

Forage à sec,

$\emptyset 311 \mathrm{~mm}$.

profondeur $300 \mathrm{~m}$ à déplacement vertical Embrayage

4 colonnes

Etanchéité

Appui de la machine Galerie à $750 \mathrm{~m}$

Cave

Tubage

Ciment

Forage élargi

Forage

Outil

Entrée d'air

Entraînement hydraulique Sortie d'air et de sel broyé Principe de l'installation de forage à sec 